\title{
On the Cascading Spectrum Contention Problem in Self-coexistence of Cognitive Radio Networks
}

\author{
Lin Chen*, Kaigui Bian*, Lin Chen ${ }^{\dagger}$, Wei Yan*, and Xiaoming Li* \\ *Institute of Network Computing and Information Systems, School of EECS, Peking University, Beijing, \\ China 100871. \\ †Lab de Recherche Informatique (LRI-CNRS UMR 8623), University Paris-Sud, 91405 Orsay, France. \\ *\{abratchen, bkg, w, Ixm\}@pku.edu.cn, ${ }^{\dagger}$ chen@Iri.fr
}

\begin{abstract}
In cognitive radio $(\mathrm{CR})$ networks, the coexistence between neighboring secondary networks is referred to as self coexistence. As prescribed in IEEE 802.22, a self-coexistence protocol enables a CR network in need of more spectrum resources to acquire spectrum (channels) from neighboring CR networks via a distributed inter-network spectrum contention process. A network that forfeits part of its spectrum in a spectrum contention process may later become short of spectrum, and in turn, it initiates a cascading spectrum contention process to acquire more spectrum resources. As a result, a local spectrum contention may trigger a series of successive contention instances that proliferate over the whole network, which may waste the network resources. In this paper, we systematically study the cascading spectrum contention problem using a percolation-based model in the context of CR networks. We show that cascading spectrum contentions under existing spectrum contention resolution rules is equivalent to a site percolation process that can readily lead to a network-wide cascade. To address such a problem, we identify the critical conditions for determining the occurrence of cascading spectrum contentions, and propose a biased spectrum contention protocol that intentionally lowers the probability that a starving network can trigger successive spectrum contentions. We show that the proposed solution can effectively restrict the spatial cascading impact of contentions.
\end{abstract}

\section{Categories and Subject Descriptors}

C.2.2 [Computer-Communication Networks]: Network Protocols

\section{Keywords}

Dynamic spectrum access, self coexistence, percolation, cognitive radio

\section{INTRODUCTION}

Permission to make digital or hard copies of all or part of this work for personal or classroom use is granted without fee provided that copies are not made or distributed for profit or commercial advantage and that copies bear this notice and the full citation on the first page. Copyrights for components of this work owned by others than ACM must be honored. Abstracting with credit is permitted. To copy otherwise, or republish, to post on servers or to redistribute to lists, requires prior specific permission and/or a fee. Request permissions from permissions@ acm.org.

$C R A B$ '13, October 4, 2013, Miami, Florida, USA

Copyright 2013 ACM 978-1-4503-2368-0/13/10 ...\$15.00.

http://dx.doi.org/10.1145/2508478.2508479.
In cognitive radio (CR) networks, the coexistence between unlicensed (secondary) users that form CR networks and licensed (primary) users is referred to as incumbent coexistence. Meanwhile, CRs have the capability to alleviate problems related to self-coexistence, the coexistence between wireless systems of the same type, such as spectrum sharing among neighboring IEEE 802.22 CR networks [12]. An 802.22 network (cell) is an infrastructure-based CR network and composed of a Base Station (BS) and several user devices (i.e., Consumer Premise Equipments). Incumbent services in IEEE 802.22 refer to TV broadcasting services or services for Part 74 devices (wireless microphones) operating in TV bands ${ }^{1}$, and secondary users refer to IEEE 802.22 entities (BS and user devices).

A self-coexistence protocol facilitates the dynamic spectrum sharing among coexisting networks in a distributed manner when a network is in need of spectrum to satisfy its service requirement. There is no need to start the spectrum sharing process for self-coexistence when the available spectrum is sufficient to satisfy all coexisting networks.

When the available spectrum is insufficient, every CR network (or network BS) occupies an amount of spectrum that is no more than it needs (i.e., its service requirement). IEEE 802.22 defines an inter-BS spectrum contention protocol for network cells to achieve the goal of self-coexistence.

- A BS that is in need of spectrum (contention source $\mathrm{BS}$ ) is allowed to win channels via pairwise contentions with its neighboring BSs (contention destination $\mathrm{BSs}$ ).

- If the contention source wins the contention, it occupies the contended channels exclusively, while the contention destinations vacate those channels via channel switching.

To ensure the fairness in the contention process, existing proposals adopt a simple unbiased contention resolution rule based on random number selection $[10,8,9,5,6]$, such that either a contention source or a contention destination has an equal probability of winning the pairwise contention.

However, the existing design of a self-coexistence protocol fails to consider the successive events that may be triggered by the spectrum redistribution during a local spectrum contention process. For example, the channel redistribution via contentions may satisfy the contention source, but meanwhile the contention destination that loses spectrum may

\footnotetext{
${ }^{1}$ Part 74 devices are small-scale, low-power wireless devices, such as wireless microphones [12, 13], which are licensed to operate in the TV broadcast bands.
} 
become short of spectrum and successively initiate a cascading spectrum contention process to acquire more spectrums. $^{2}$ As a result, unrestricted local spectrum contentions may trigger a series of successive contention instances that proliferate over the whole network, which may waste the network resources.

In this paper, we systematically study the cascading spectrum contention problem using the percolation theory in the context of CR networks. We show that cascading spectrum contentions under existing spectrum contention resolution rules is equivalent to a site percolation process that can readily lead to a network-wide cascade. Moreover, we have found the critical conditions for determining the occurrence of cascading spectrum contentions, and propose a biased spectrum contention protocol to mitigate this problem.

The rest of this paper is organized as follows: we provide technical background for IEEE 802.22 and percolation theory in Section 2. We formulate the problem in Section 3, and show the feasibility of cascading spectrum contentions in CR networks in Section 4. We propose a biased spectrum contention protocol that mitigates the problem in Section 5. In Section 6 , we compare different contention resolution rules using simulation results. We conclude the paper in Section 7.

\section{TECHNICAL BACKGROUND}

\subsection{Spectrum Contention for Self-coexistence}

IEEE 802.22 is the first and only wireless standard based on CR technology, which provides the broadband access in rural areas. Every 802.22 network is under control of a central entity, i.e., a Base Station (BS). We will use the 802.22 network as an example of a CR network in the forthcoming discussions.

Inter-BS communication. Two co-located BSs in neighboring cells are called neighboring BSs. Inter-BS communication between neighboring BSs can be well supported in 802.22 , via either inter-BS control message exchange overthe-air, or over the backhaul control (wired) connections $[6$, 8].

Spectrum contention. When available spectrum is insufficient to satisfy all coexisting BSs, an 802.22 BS in need of spectrum can initiate an inter- $B S$ spectrum contention process so that better channels or more channels can be acquired from neighboring BSs to satisfy the QoS of its workload [12].

1. The BS that initiates the spectrum contention process is the contention source ( $S R C)$ ). A spectrum contention process consists of a number of pairwise contentions, and every pairwise contention is carried out between the SRC and a neighboring BS that is referred to as the contention destinations (DST).

2. The SRC sends a contention request message to contend for a target channel that is currently occupied by a DST. The DST uses a specific contention resolution rule to determine the winner of the contention.

3. In the unbiased contention resolution rule $[10,8,9,5$, 6], every BS (either SRC or DST) is required to select a Spectrum Contention Number (SCN) that is uniformly

\footnotetext{
${ }^{2} \mathrm{~A}$ cascade is a series of events, in which the occurrence of
} an event can trigger the occurrence of successive events. distributed in the range $[0, W-1]$, and exchange the SCN values, where $W$ is a constant representing the contention window size.

4. The BS that has selected the largest $\mathrm{CN}$ among all participating $\mathrm{BSs}$ is the winner of the contention. Other BSs (and their 802.22 networks) that fail to win will vacate the channel.

\subsection{Site Percolation}

A percolation process resides in a graph including sites (vertices) or bonds (edges). The most common percolation model takes the graph structure of a regular lattice (e.g., a square lattice). In the site percolation process, every site is either open (i.e., open to flow, diffusion, etc.) randomly and independently with probability $p$, or closed (i.e., closed to flow, diffusion, etc.) with probability $1-p$.

Definition 1. A path is open if all its sites are open and it is close if all its sites are closed. Sites $u$ and $v$ are said to be open connected if there exists an open path that connects $u$ and $v$. We define $v$ to be open connected to itself.

It follows immediately that open connection is an equivalence relation. We write $u \leftrightarrow v$ if $u$ and $v$ are open connected, and $u \leftrightarrow v$ if $u$ and $v$ are not open connected.

Definition 2. The open cluster $C(v)$ at site $v$ is the set of all sites that are open connected to $v$, represented as

$$
C(v)=\{u \in V \mid u \leftrightarrow v\} .
$$

Intuitively, as $p$ increases, the size of an open cluster also increases. At a critical value of $p$, the long-range connectivity in the network appears - there is a transition in the topological structure of the network from a macroscopically disconnected to a connected one - and thus this critical value is called the percolation threshold or critical probability [7]. Let $p_{c}$ denote the percolation threshold, we have the following fundamental results from percolation theory [7]:

- when $p>p_{c}$, with probability one, there exists an infinite cluster; and with a positive probability, the origin (or any other fixed point) belongs to an infinite cluster;

- when $p<p_{c}$, all clusters are finite.

When the graph structure resides in continuous space (e.g., a random geometric graph), the resulting percolation model is described as continuum percolation. Continuum percolation theory has served as a useful tool in many applications in wireless networks, such as power management [14], latency evaluation $[3,16,15]$, connectivity analysis[2], node failure detection [17], etc.

\section{A PERCOLATION-BASED FRAMEWORK}

\subsection{System Model}

Network graph. The placement of BSs of CR networks could transform to an undirected network graph $G=(V, E)$, where $V$ is the set of vertices and $E$ is the set of edges. Each vertex $i \in V$ represents a BS of a network cell, and we call the BS represented by a vertex $i$ as BS $i$. If two BSs $i$ and $j$ are neighboring to each other in the network, there is an edge $\{i, j\} \in E$ connecting the two vertices $i, j \in V$ (i.e., an 
inter-BS communication link connecting the two BSs). In this case, vertex $j$ is said to be a neighbor of vertex $i$. Let $N(i)$ denote the set of all neighbors of vertex $i$ in graph $G$ : $N(i)=\{j \in V \mid\{i, j\} \in E\}$. The cardinality of $N(i)$ is called the degree of vertex $i$, written as $d(i)=|N(i)|$.

Base station placement on a lattice. In an 802.22 system, the rural area is divided into regular shaped cells, which can be hexagonal, square, or some other irregular shapes. We generalize them to the notion of lattice [1], and three common types of lattices are triangular, square and honeycomb lattices.

Service requirement Every BS $i$ requires $r_{i}$ channels to satisfy the QoS of its admitted workload, and $N$ is the maximum number of available channels. The value of $r_{i}$, called the service requirement of $B S i$, depends on the intracell traffic demand raised by the secondary users (i.e., CPEs) connected to the BS $i$. Let $A_{i}$ denote the set of channels that are occupied by BS $i$.

Every BS $i$ tries to claim as many unoccupied channels as possible until $\left|A_{i}\right|=r_{i}$ or there is no unoccupied channels that can be claimed. Thus, $\left|A_{i}\right| \leq r_{i}$ for any BS $i$. To avoid co-channel interference, neighboring BSs $i$ and $j$ occupy disjoint sets of channels, i.e., $A_{i} \cap A_{j}=\varnothing$.

Network states. Every BS $i$ occupies an amount of spectrum that is no more than its service requirement. We assume there are two states for a given CR network - a state wherein the BS is in need of spectrum, and a state wherein the BS does not need additional spectrum. We refer these two states as "starving" and "satisfied", respectively.

- When $\left|A_{i}\right|<r_{i}$, we call BS $i$ a starving $B S$.

- When $\left|A_{i}\right|=r_{i}$, we call BS $i$ a satisfied $B S$.

Causes for spectrum contention. The root cause for incurring spectrum contention is the existence of a starving BS. There are three factors that make a satisfied BS $i$ become starving: (1) the reclaim of occupied channels in $A_{i}$ by the primary user; (2) the increase of $r_{i}$ due to an increased intra-cell workload; and (3) losing channels in $A_{i}$ due to spectrum contentions.

We call the probability that a satisfied BS $i$ becomes starving due to all these factors as the starving probability of BS $i$, denoted by $p_{i}$. Meanwhile, we call the probability that a satisfied BS $i$ becomes starving due to non-contention (the first two) factors as spontaneous starving probability, denoted by $p_{i, 0}$.

\subsection{Problem Formulation}

In an inter-BS spectrum contention process, the channel redistribution may satisfy the contention source BS $i$; but meanwhile a contention destination BS $j$ that loses the target channel may become starving, and successively initiate a cascading contention process. Therefore, the event that a BS $j$ becomes starving is caused by a spectrum contention initiated by a starving BS $i$. That is, a local spectrum contention initiated by a BS may cause a cascade of spectrum contentions, which will result in futile contention results and waste network resources. We refer to such a phenomenon as a cascading spectrum contention.

We formulate the phenomenon of cascading spectrum contentions as a site percolation process over the network graph as follows.
Similar to the definitions of open/closed sites (vertices) in the percolation process, we define open/closed BSs in the context of CR networks.

Definition 3. A vertex $i$ in the network graph $G$ is open if $B S i$ is a starving $B S$, and we call it an open BS. Otherwise, the vertex $i$ is closed if $B S i$ is a satisfied $B S$, and we call it a closed $B S$.

Definition 4. Two BSs $i$ and $j$ are said to be open connected if there exists a path in the network graph that connects vertices $i$ and $j$, and every vertex in this path is open. The open cluster at BS $i$ is the set of all BSs that are open connected to $B S i$.

It is believed that BSs $i$ and $j$ in the same open cluster are related in a certain relationship of spectrum contentions, e.g., there may exist a path starting at BS $i$ and ending at BS $j$, where a pairwise contention occurs between every pair of BSs along this path, or there exist two contention paths between $k$ and $i, k$ and $j$, where $k$ is a third BS in the same cluster. The open cluster in the network graph describes the set of BSs that are in the "starving" state that may be caused by cascading spectrum contentions.

The size of an open cluster. We refer to metrics used in the percolation theory to quantify the magnitude of cascading spectrum contentions. We define the mean open cluster size at BS $i$ as

$$
\chi_{i}\left(p_{i}: i \in V\right)=E_{\left(p_{i}: i \in V\right)}(|C(i)|),
$$

where $E_{\left(p_{i}: i \in V\right)}(X)$ denotes the expectation of a random variable $X$, given that $\mathrm{BS} i$ is open independently with probability $p_{i}(i \in V)$.

Lower bound case with starving probability $p$. In Section 4.1, we introduce a lower bound $p$ of $p_{i}$ 's, i.e. $p \leq p_{i}$, $\forall i \in V$. Thus $\chi_{i}\left(p_{i}: i \in V\right) \geq \chi_{i}(p) \triangleq E_{p}(|C(i)|)$, where $E_{p}(X)$ denotes the expectation given that every BS is open independently with probability $p$, i.e. $\chi_{i}(p)$ is a lower bound of $\chi_{i}\left(p_{i}: i \in V\right)$.

So far we have transformed the study of $\chi_{i}\left(p_{i}: i \in V\right)$ into the study of $\chi_{i}(p)$ in a lower bound case where every BS $i$ is open independently with probability $p$.

Since the placement of BSs of CR networks form a lattice $G=(V, E)$, whose automorphism group acts transitively upon $V$ (also known as vertex-transitive) [1], then $\forall i, j \in V$, $C(i)=C(j)$ and $\chi_{i}(p)=\chi_{j}(p)$ due to the homogeneity of a lattice. Hence, we simply use $C$ and $\chi(p)$ instead of $C(i)$ and $\chi_{i}(p)$.

Therefore, the cascading spectrum contention process in CR networks is mapped to the lower bound site percolation process over the network graph where every vertex (BS) is open independently with probability $p$.

\subsection{Global and Severe Cascades}

Since $\chi(p)$ is defined to characterize the magnitude of cascading spectrum contentions, a global cascade of spectrum contentions occurs if the mean open cluster size is infinite, i.e., $\chi(p)=\infty$. According to the percolation theory, an infinite open cluster exists $(\chi(p)=\infty)$ with probability one, if and only if $p \geq p_{c}$, where $p$ is the starving probability and $p_{c}$ is the critical probability.

In the subcritical phase when $p<p_{c}$, a severe cascade of spectrum contentions is to said to occur if the mean open cluster size $\chi(p) \geq \chi(\chi$ is a predefined threshold, e.g., that 


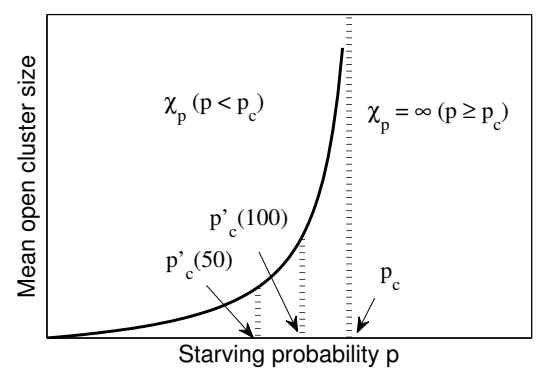

Figure 1: The mean open cluster size $\chi(p)$ vs. the starving probability. The (modified) critical probabilities are marked, and $p_{c}^{\prime}(50)<p_{c}^{\prime}(100)<p_{c}$.

$\chi$ is set to be 50 means that a cascade involving in over 50 BSs is considered to be a severe cascade), which suggests that an average open cluster of BSs is large.

In Theorem 3, we will show that there exists a modified critical probability, $p_{c}^{\prime}(\chi)$, such that if $p>p_{c}^{\prime}$, a severe cascade with $\chi(p) \geq \chi$ occurs. We roughly illustrate the critical probability $p_{c}$ and two modified critical probabilities $p_{c}^{\prime}(50)$ and $p_{c}^{\prime}(100)$ in Figure 1.

\section{MAIN RESULTS}

In this section, we present analytical results on the mean open cluster size, and the criteria for determining the existence of a global or severe cascade in CR networks.

\subsection{Lower Bound of Starving Probability}

Four parameters related to the lower bound starving probability $p$. We introduce four parameters that define the behaviors of coexisting networks in a spectrum contention process, which will collectively affect the size of an open cluster. If we can establish the relationship between $p$ and these parameters, we will be able to analyze $\chi(p)$ and $\chi_{i}\left(p_{i}: i \in V\right)$ based on these parameters. The four parameters are described as follows.

1. $d \in \mathbb{N}$, the degree of each vertex. Note that $d=6$ in a triangular lattice, $d=4$ in a square lattice and $d=3$ in a honeycomb lattice.

2. $k \in \mathbb{N} \cap[0, d]$, the number of pairwise contentions initiated by a contention source in each spectrum contention process. If $k=0, \mathrm{BSs}$ are in fact non-collaborative, reluctant to be involved in a channel transaction. If $k>0$, they are collaborative. In particular, when $k=d$, the contention source initiates pairwise contentions with all of its neighboring BSs.

3. $\lambda \in[0,1]$, the winning probability of the contention source in a pairwise contention: In an unbiased contention resolution rule, $\lambda=1 / 2$; In a biased contention resolution rule, $\lambda$ is not necessarily $1 / 2$;

4. $p_{0}=\inf _{i \in V} p_{i, 0} \in[0,1]$, the minimum probability that a BS becomes starving spontaneously rather than due to spectrum contentions.

Then, we define the following three events:

- Event $S^{(i)}$ : a given BS $i$ is starving;
- Event $S_{s}^{(i)}$ : a given BS $i$ is starving spontaneously;

- Event $S_{c}^{(i)}$ : a given BS $i$ is starving due to spectrum contention initiated by its neighbors.

Events $S_{s}^{(i)}$ and $S_{c}^{(i)}$ are assumed to be independent.

We denote the event that BS $j$ wins a pairwise contention over $\mathrm{BS} i$ by $W_{j, i}$. For BS $j$ that is a neighbor of $\mathrm{BS} i$, $P\left(W_{j, i}\right)$ is the probability that BS $j$ is starving, which is at least $p_{0}=\inf _{i \in V} p_{i, 0}$, times the probability that BS $j$ selects BS $i$ when BS $j$ attempts to select $k$ out of $d$ neighbors of its to initiate a pairwise spectrum contention since it is starving at the moment, times the probability that BS $j$ wins the pairwise spectrum contention with BS $i$. Therefore, $P\left(W_{j, i}\right) \geq p_{0} \lambda k / d$. Then, we derive the probability of event $S_{c}^{(i)}$ as follows.

$$
\begin{aligned}
P\left(S_{c}^{(i)}\right) & =P\left(\bigcup_{j \in N(i)} W_{j, i}\right)=1-P\left(\bigcap_{j \in N(i)} W_{j, i}^{c}\right) \\
& =1-\prod_{j \in N(i)} P\left(W_{j, i}^{c}\right)=1-\prod_{j \in N(i)}\left(1-P\left(W_{j, i}\right)\right) \\
& \geq 1-\prod_{j \in N(i)}\left(1-\frac{p_{0} \lambda k}{d}\right) \\
& =1-\left(1-\frac{p_{0} \lambda k}{d}\right)^{d} .
\end{aligned}
$$

Since $P\left(S_{c}^{(i)}\right) \geq 1-\left(1-\frac{p_{0} \lambda k}{d}\right)^{d}$, we have the lower bound for $p_{i}$ such as:

$$
\begin{aligned}
p_{i}=p_{i}\left(p_{i, 0}, d, k, \lambda\right) & =P\left(S^{(i)}\right)=P\left(S_{s}^{(i)} \cup S_{c}^{(i)}\right) \\
& =1-\left(1-P\left(S_{s}^{(i)}\right)\right)\left(1-P\left(S_{c}^{(i)}\right)\right) \\
& \geq 1-\left(1-p_{0}\right)\left(1-\frac{p_{0} \lambda k}{d}\right)^{d}
\end{aligned}
$$

Let

$$
p=l\left(p_{0}, d, k, \lambda\right) \triangleq 1-\left(1-p_{0}\right)\left(1-\frac{p_{0} \lambda k}{d}\right)^{d} \leq p_{i} .
$$

Properties of the lower bound function. We intuitively expect the following properties of the lower bound function $p=l\left(p_{0}, d, k, \lambda\right)$.

- The increase of $p_{0}, k$ and $\lambda$ intuitively galvanizes the spectrum contention and makes a BS more likely to be starving. Therefore, $p$ is a strictly increasing function with regard to $p_{0}, k$ and $\lambda$.

- When $p_{0}=1$, every BS becomes starving with probability one, i.e. $p_{i}=p=1, \forall i \in V$.

- Whenever $d=0, k=0$ or $\lambda=0$, it reduces to the case when every BS is non-collaborative and there is no spectrum contention initiated at all. In this case, we have $p=p_{0}$, i.e., the probability that $\mathrm{BS} i$ is starving is exactly the spontaneous starving probability.

In Theorem 1 , we show that the function $l$ satisfies the above properties.

Theorem 1. For $0 \leq p_{0} \leq 1, d \in \mathbb{N}$ and $k \in \mathbb{N} \cap[0, d]$,

1. $0 \leq p_{0} \leq l\left(p_{0}, d, k, \lambda\right) \leq 1$.

2. $l\left(p_{0}, d, k, \lambda\right)$ is a strictly increasing function with regard to $p_{0}, k$ and $\lambda$. 
3. $l\left(p_{0}, d, k, \lambda\right)$ is a strictly decreasing function with regard to $d$. Fix $k / d=r, l\left(p_{0}, d, k, \lambda\right)=l\left(p_{0}, d, r d, \lambda\right)$ is a strictly increasing function with regard to $d$.

4. $l(1, d, k, \lambda)=1$.

5. $\lim _{d \rightarrow 0+0} l\left(p_{0}, d, k, \lambda\right)=p_{0}, l\left(p_{0}, d, 0, \lambda\right)=p_{0}$, and $l\left(p_{0}, d, k, 0\right)=p_{0}$.

The proof of Theorem 1 only involves in easy calculus.

\subsection{Mean Open Cluster Size}

The probability of the occurrence of a global or severe cascade of spectrum contentions is equivalent to the probability that an infinite/large open cluster exists in the network graph.

Based on the results in [1], Theorem 2 shows how fast $\chi(p)$ increases in the subcritical phase $\left(p<p_{c}\right)$

Theorem 2. $G$ is a lattice, then for $p \in\left[0, p_{c}\right)$

$$
\chi(p) \geq \underline{\chi}(p),
$$

where

$$
\underline{\chi}(p)=\frac{p p_{c}}{\left|p_{c}-p\right|}
$$

and for $p \in\left[p_{c}, 1\right]$,

$$
\chi(p)=\infty .
$$

Equation (1) tells the relationship between $\chi(p)$ and $p$ in the subcritical phase. Since $\chi_{i}\left(p_{i}: i \in V\right) \geq \chi(p) \geq \chi(p)$, it is feasible to study $\chi_{i}\left(p_{i}: i \in V\right)$ via $\underline{\chi}(p)$ and identify conditions for the occurrence of global or severe cascades.

\subsection{Conditions for the Occurrence of Global or Severe Cascades}

Now we consider the lower bound case. If $\chi(p)=\infty$, a global cascade of spectrum contentions occurs; and if $\chi(p) \geq$ $\chi$ ( $\chi$ is a predefined threshold), a severe cascade occurs. We are interested in the following two problems:

1. Under what conditions a global cascade of spectrum contention occurs;

2. Under what conditions a severe cascade occurs.

Theorem 3 gives a criterion for determining the occurrence of global or severe cascades.

Theorem 3. Suppose $G$ is a lattice.

1. If $l\left(p_{0}, d, k, \lambda\right) \geq p_{c}$, the spectrum contention protocol induces a global cascade of spectrum contentions with probability one,

2. If

$$
l\left(p_{0}, d, k, \lambda\right) \geq\left(\frac{1}{p_{c}}+\frac{1}{\chi}\right)^{-1} \triangleq p_{c}^{\prime}(\chi),
$$

where $p_{c}^{\prime}$ is the modified critical probability, then the mean open cluster size $\chi(p) \geq \chi$.

Proof. If $l\left(p_{0}, d, k, \lambda\right) \geq p_{c}$, we have

$$
p \geq l\left(p_{0}, d, k, \lambda\right) \geq p_{c} .
$$

By Theorem 2, $\chi(p)=\infty$, i.e., it induces a global cascade of spectrum contentions with probability 1 .
If $p<p_{c}$ and

$$
p \geq l\left(p_{0}, d, k, \lambda\right) \geq p_{c}^{\prime}=\left(\frac{1}{p_{c}}+\frac{1}{\chi}\right)^{-1},
$$

we have

$$
\chi(p) \geq \underline{\chi}(p)=\frac{p p_{c}}{p_{c}-p} \geq \chi,
$$

i.e. it induces the mean open cluster size $\chi(p)$ to be $\chi(p) \geq$ $\chi$.

If $p \geq p_{c}$, we directly have

$$
\chi(p)=\infty>\chi
$$

by Theorem 2 . This completes the proof.

We notice that if $\chi=\infty$, then $p_{c}^{\prime}=p_{c}$, and hence the first clause of Theorem 3 is just a special case of the second one. The occurrence of a global cascade (an infinite open cluster) is simply the case of $\chi=\infty$.

Corollaries 1 and 2 show that given a specific graph structure (i.e. a fixed $d$ ), there also exists a threshold such that: if $p_{0}$ (or $k$ ) exceeds the threshold, a global or severe cascade occurs.

Corollary 1. Fix $d \in \mathbb{N}$ and $k \in \mathbb{N} \cap[0, d]$,

1. There exists a threshold $p_{0 ; c} \in[0,1]$ such that if $p_{0}>$ $p_{0 ; c}$, then a spectrum contention protocol induces the mean open cluster size $\chi(p)$ to be $\chi(p) \geq \chi$. In particular, if $p_{0}>p_{c}^{\prime}, \chi(p) \geq \chi$, where $p_{c}^{\prime}$ is the modified critical probability with respect to $\chi$.

2. There exists a threshold $\tilde{p}_{0 ; c} \in[0,1]$ such that if $p_{0}>$ $p_{0 ; c}$, then a spectrum contention protocol induces a glocal cascade of spectrum contentions with probability 1 . In particular, if $p_{0}>p_{c}$, a global cascade occurs.

Proof. Take $p_{0 ; c}=l^{-1}\left(p_{c}^{\prime}\right) \in[0,1]$, where $l^{-1}(p)$ is the inverse function of $l\left(p_{0}\right)=l\left(p_{0}, d, k, \lambda\right)$. Since $l(0)=0$, $l(1)=1$ and $l\left(p_{0}\right)$ is strictly increasing with regard to $p_{0}$, therefore such $p_{0 ; c}=l^{-1}\left(p_{c}^{\prime}\right)$ exists.

If $p_{0}>p_{0 ; c}$, we have

$$
l\left(p_{0}\right)>l\left(p_{0 ; c}\right)=p_{c}^{\prime} .
$$

And by Theorem $3, \chi(p) \geq \chi$. If $p_{0}>p_{c}^{\prime}$, by Theorem 1 , we have

$$
l\left(p_{0}\right) \geq p_{0}>p_{c}^{\prime},
$$

and hence, by Theorem 3 again, $\chi(p) \geq \chi$.

Now we take $\chi=\infty$, then $p_{c}^{\prime}=p_{c}$, and this proves the second clause.

Corollary 2. Fix $p_{0} \in[0,1]$ and $d \in \mathbb{N}$,

1. If

$$
d \geq \frac{\ln \left(1-p_{c}^{\prime}\right)-\ln \left(1-p_{0}\right)}{\ln \left(1-\lambda p_{0}\right)},
$$

then there exists a threshold $k_{c} \in[0, d]$ such that if $k>k_{c}$, then a spectrum contention protocol induces the mean open cluster size $\chi(p)$ to be $\chi(p) \geq \chi$.

2. If

$$
d \geq \frac{\ln \left(1-p_{c}\right)-\ln \left(1-p_{0}\right)}{\ln \left(1-\lambda p_{0}\right)},
$$

then there exists a threshold $\tilde{k}_{c} \in[0, d]$ such that if $k>\tilde{k}_{c}$, then a spectrum contention protocol induces a global cascade of spectrum contentions with probability one. 
Proof. Let $l(k)$ denote $l\left(p_{0}, d, k, \lambda\right)$ for short. We have $l(0)=p_{0}$ and $l(d)=1-\left(1-p_{0}\right)\left(1-\lambda p_{0}\right)^{d} \geq p_{c}^{\prime}$.

If $p_{0}>p_{c}^{\prime}$, by Corollary $1, \chi(p) \geq \chi$.

Now we assume that

$$
p_{0}=l(0) \leq p_{c}^{\prime} \leq l(d)
$$

By the strict monotonicity of $l(k), l^{-1}\left(p_{c}^{\prime}\right)$ exists and let $k_{c}$ be $k_{c}=l^{-1}\left(p_{c}^{\prime}\right) \in[0, d]$, where $l^{-1}(p)$ is the inverse function of $l(k)=l\left(p_{0}, d, k, \lambda\right)$. If $k>k_{c}$, we have

$$
l(k)>l\left(k_{c}\right)=p_{c}^{\prime} .
$$

By Theorem 3, $\chi(p) \geq \chi$.

Now we take $\chi=\infty$, then $p_{c}^{\prime}=p_{c}$, this proves the second clause.

\subsection{Applicable Criteria for Detecting Global or Severe Cascades}

Although Theorem 3 provides criteria to determine whether a global or severe cascade occurs, yet it is less applicable due to its complicated expressions.

To be more applicable for concrete calculation (for the IEEE 802.22 case), Theorem 4 gives a criterion that is easy to check. Finally, this leads us to a very simple criterion when applied to three typical types of network topology, the triangular, square and honeycomb lattices.

LEMmA 1. Given $p_{c}^{\prime} \in[0,1]$ and $d \in \mathbb{N}$, there exists a unique solution $p_{0}=\Lambda\left(p_{c}^{\prime}, d\right) \in[0,1]$ of the equation with respect to $p_{0}$

$$
l\left(p_{0}, d, d, \lambda\right)=p_{c}^{\prime} .
$$

In addition, $\Lambda \leq p_{c}^{\prime}$.

Proof. By Theorem $1, l\left(p_{0}\right)=l\left(p_{0}, d, k, \lambda\right)$ is a strictly increasing continuous function with regard to $p_{0} \in[0,1]$, and $l(0)=0, l(1)=1$. This implies a unique solution of the eqation above.

By Theorem $1, p_{c}^{\prime}=l(\Lambda, d, d, \lambda) \geq \Lambda$.

Theorem 4 gives a linear criterion (see Equation (3)), which is easier to check and applicable in practical network topologies.

Theorem 4. $G$ is a lattice with vertex degree $d$. A spectrum contention protocol induces the mean open cluster size $\chi(p)$ to be $\chi(p) \geq \chi$ if

$$
p_{0}+B k \geq p_{c}^{\prime},
$$

where $p_{c}^{\prime}$ is the modified critical probability and $B=\left(p_{c}^{\prime}-\right.$ $\Lambda) / d$ is a constant. In particular, if we take $\chi=\infty$, it actually occurs a global cascade of spectrum contentions.

PROOF. If $p_{0} \geq p_{c}^{\prime}$, we directly have $p \geq p_{0} \geq p_{c}^{\prime}$

$$
\chi(p) \geq \underline{\chi}(p)=\frac{p p_{c}}{p_{c}-p} \geq \chi
$$

by Theorem 2 . Now we only consider the case where $p_{0}<p_{c}^{\prime}$. If we have

$$
k \geq \kappa\left(p_{0}\right)=\frac{d}{\lambda p_{0}}\left(1-\sqrt[d]{\frac{1-p_{c}^{\prime}}{1-p_{0}}}\right),
$$

then using the fact $0 \leq p_{0}<p_{c}^{\prime}$, we will have

$$
l\left(p_{0}, d, k, \lambda\right) \geq p_{c}^{\prime},
$$

and by Theorem 3, a spectrum contention protocol induces the mean open cluster size $\chi(p)$ to be $\chi(p) \geq \chi$. Hence it suffices to show that $k \geq \kappa\left(p_{0}\right)$.

If $p_{0}<\Lambda$, we have

$$
\begin{aligned}
p_{0}+B k & <\Lambda+B k \\
& \leq \Lambda+B d \\
& =\Lambda+p_{c}^{\prime}-\Lambda \\
& =p_{c}^{\prime} .
\end{aligned}
$$

Since $p_{0}+B k \geq p_{c}^{\prime}$, we have $p_{0} \geq \Lambda$.

By the definition of $\Lambda, \kappa(\Lambda)=d$. In addition, $\kappa\left(p_{c}^{\prime}\right)=0$. It is easy to check that $\kappa^{\prime}\left(p_{0}\right)<0$ and $\kappa^{\prime \prime}\left(p_{0}\right)>0$ on $\left[\Lambda, p_{c}^{\prime}\right]$, therefore $\forall p_{0} \in\left[\Lambda, p_{c}^{\prime}\right]$,

$$
\kappa\left(p_{0}\right) \leq \frac{d}{\Lambda-p_{c}^{\prime}}\left(p_{0}-p_{c}^{\prime}\right) .
$$

Since $p_{0}+B k \geq p_{c}^{\prime}$, we have

$$
\begin{aligned}
k & \geq \frac{p_{c}^{\prime}-p_{0}}{B} \\
& =\frac{d\left(p_{0}-p_{c}^{\prime}\right)}{\Lambda-p_{c}^{\prime}} \\
& \geq \kappa\left(p_{0}\right) .
\end{aligned}
$$

This completes the proof.

Equation (3) can be rewritten as

$$
k+C_{0}\left(\chi, p_{c}, d, \Lambda\right) p_{0} \geq C_{1}\left(\chi, p_{c}, d, \Lambda\right),
$$

where $C_{0}=d\left(\chi+p_{c}\right)\left(\chi p_{c}-\Lambda\left(\chi+p_{c}\right)\right)^{-1}$ and $C_{1}=d \chi p_{c}\left(\chi p_{c}-\right.$ $\left.\Lambda\left(\chi+p_{c}\right)\right)^{-1}$.

By Theorem 4, we give very clear criteria to detect the occurrence of a global or severe cascade in three typical lattice structures.

THEOREM 5. Suppose IEEE 802.22 contention resolution protocol is used, and $\lambda=1 / 2$,

1. In the triangular lattice case, $d=6, p_{c}=1 / 2$ and $\Lambda=\Lambda_{t}=0.164151$. If $k+C_{0}(\chi, 1 / 2,6,0.164151) p_{0} \geq$ $C_{1}(\chi, 1 / 2,6,0.164151)$, the mean open cluster size will be at least $\chi$. In particular, if

$$
k+17.8652 p_{0} \geq 8.9326,
$$

a global cascade occurs.

2. In the square lattice case, $d=4, p_{c}=0.5927$ and $\Lambda=$ $\Lambda_{s}=0.213907$. If $k+C_{0}(\chi, 0.5927,4,0.213907) p_{0} \geq$ $C_{1}(\chi, 0.5927,4,0.213907)$, the mean open cluster size will be at least $\chi$. In particular, if

$$
k+10.5599 p_{0} \geq 6.2588,
$$

a global cascade occurs.

3. In the honeycomb lattice case, $d=3, p_{c}=0.6970$ and $\Lambda=\Lambda_{h}=0.251597$. If $k+C_{0}(\chi, 0.6970,3,0.251597) p_{0} \geq$ $C_{1}(\chi, 0.6970,3,0.251597)$, the mean open cluster size will be at least $\chi$. In particular, if

$$
k+6.7355 p_{0} \geq 4.6946,
$$

a global cascade occurs. 
Proof. Since Equation (4) is a rewritten version of Equation (3), by Theorem 4, this completes the proof. Now we take the triangular lattice case as an example of numerical calculation. The first step is to calculate $\Lambda$. Since $d=6, \lambda=1 / 2, p_{c}^{\prime}=p_{c}=1 / 2$ in Equation (2), we have $1-\left(1-p_{0}\right)\left(1-p_{0} / 2\right)^{6}=p_{0}$ and obtain $p_{0}=0.164151$. Therefore $\Lambda=p_{0}=0.164151$. When $\chi=100$, we have $C_{0}=17.9985$ and $C_{1}=8.9545$. If $k+17.9985 p_{0} \geq 8.9545$, a cascade of spectrum contentions with $\chi \geq 100$ occurs. When $\chi=\infty$, we have $C_{0}=17.8652$ and $C_{1}=8.9326$. If $k+17.8652 p_{0} \geq 8.9326$, a global cascade occurs.

\section{A BIASED SPECTRUM CONTENTION PROTOCOL}

\subsection{Contention Resolution Rule}

We propose a biased contention resolution rule that mitigates this problem by reducing the winning probability of a contention source in a pairwise contention. We define a contention path between BSs $i$ and $j$ as a path between vertices $i$ and $j$ in the network graph, such that the channel redistribution via a pairwise contention process occurs for every pair of neighboring BSs that belong to the path. The procedure for the biased contention resolution is described below.

1. In the contention request, every contention source BS $i$ includes the target channel number $h$, its SCN $s_{i}$ chosen from $[0, W-1]$, and the current length of the contention path $l_{i}$ measured by BS $i$. If the BS $i$ does not belong to any contention path, it sets $l_{i}=0$, which implies that it is the starting vertex of a new contention path.

2. Every contention destination BS $j$ checks the values of $l_{i}$ and SCN $s_{i}$ in the contention request from the contention source BS $i$. Let $S(j)$ denote the set of contention sources that send contention requests to BS $j$ during a self-coexistence window.

3. If $|S(j)|>1$, BS $j$ is being reached by more than one contention paths. The contention destination BS $j$ measures its $l_{j}$ as $\max _{i \in S(j)}\left\{l_{i}\right\}+1$, and generates its own $\mathrm{SCN} s_{j}$ from a modified contention window $\left[0, l_{j}\right.$. $W-1]$. The measured value of $l_{j}$ will be used by BS $j$ in future contention requests if it becomes a contention source.

4. If the contention destination $\mathrm{BS} j$ has the greatest $\mathrm{SCN}$ value, it wins the contention. Otherwise, the contention source who has the greatest SCN value wins, and the contention destination $\mathrm{BS} j$ releases the target channel.

If $p_{0} \geq p_{c}$, a global cascade of spectrum contentions is inevitable. The fact that $p_{0} \geq p_{c}$ strongly suggests the insufficiency of overall spectrum resources. Next, we discuss the case when $p_{i, 0}<p_{c}$ for all $i \in V$.

\subsection{Finite Cluster Size}

Decreasing the winning probability of a contention source can prevent the occurrence of infinite contention paths, which is shown in the following theorem.
THEOREM 6. There is no infinite contention path if the biased contention resolution rule is used for contention resolution in the case of $p_{i, 0}<p_{c}, \forall i \in V$.

Proof. We prove this theorem by contradiction. Suppose there is an infinite contention path $\mathcal{P}$ generated in the network. $\mathcal{P}$ is denoted as the sequence

$$
i_{0},\left\{i_{0}, i_{1}\right\}, i_{1},\left\{i_{1}, i_{2}\right\}, i_{2},\left\{i_{2}, i_{3}\right\}, i_{3}, \ldots
$$

And we have $\left\{l_{i_{n}}\right\}_{n \geq 0}$ is a strictly increasing sequence, or more precisely, $l_{i_{n+1}} \geq l_{i_{n}}+1$ for all $n \geq 0$, and therefore $\lim _{n \rightarrow \infty} l_{i_{n}}=\infty$. We write $\lambda_{u, v}$ for the winning probability of BS $u$ as a contention source and BS $v$ is the contention destination. We have

$$
\begin{gathered}
\lambda_{u, v}+\lambda_{v, u}=1 \\
\lambda_{i_{j}, i_{j+1}}=\frac{1}{2 l_{i_{j+1}}}=\frac{1}{2 \max _{v \in S\left(i_{j+1}\right)}\left\{l_{v}\right\}} \leq \frac{1}{2\left(l_{i_{j}}+1\right)},
\end{gathered}
$$

and

$$
\lambda_{i_{j+1}, i_{j}}=1-\lambda_{i_{j}, i_{j+1}} \geq 1-\frac{1}{2\left(l_{i_{j}}+1\right)} .
$$

And it is easy to see that for all $j^{\prime} \in S(j), \lambda_{j, j^{\prime}}$ is the same by the description of the biased contention resolution rule, therefore $\lambda_{j, j^{\prime}}$ is simply denoted as $\lambda_{j}$, where BS $j$ is the contention destination and $j^{\prime} \in S(j)$. By Equation (5),

$$
\lambda_{i_{j+1}} \geq 1-\frac{1}{2\left(l_{i_{j}}+1\right)},
$$

therefore $\lim _{j \rightarrow \infty} \lambda_{i_{j}}=1$. We write $p_{u}$ for the starving probability of BS $u, p_{u} \geq p_{u, 0}$. We have $\lim _{\lambda_{u} \rightarrow 1} p_{u}=p_{u, 0}$, i.e. as the winning probability of $\mathrm{BS} u$ as a contention destination approaches 1 , it becomes more and more resistant to contention sources, its starving probability just goes to $p_{u, 0}$, the spontaneous starving probability. Hence $\lim _{j \rightarrow \infty} p_{i_{j}}=$ $p_{u, 0}<p_{c}$. We take $\varepsilon_{0}$ to be $\left(p_{c}-p_{u, 0}\right) / 2$. There exists $N \in \mathbb{N}$ such that $j>N$ implies $p_{i_{j}}<p_{u, 0}+\varepsilon_{0}<p_{c}$.

By removing vertices $i_{0}, \ldots, i_{N}$ and edges that connect these vertices from the path $\mathcal{P}$, and the remaining part of $\mathcal{P}$ is still an infinite contention path $\mathcal{P}_{2}$ with the starting vertex $i_{N+1}$.

The infinite contention path $\mathcal{P}_{2}$ implies that an infinite cluster can be formed by the site percolation process in the underlying network graph $G$. Under the percolation model, $\mathcal{P}_{2}$ is an infinite cluster in $G$ and every site in $\mathcal{P}_{2}$ is open with a probability that is less than $p_{c}$. This result contradicts the fundamental fact in the percolation theory: there is no infinite cluster when the probability of an open site is less than the percolation threshold. Thus, we conclude that there is no infinite contention path if the biased contention resolution rule is used.

\section{NUMERICAL RESULTS}

In this section, we compare two contention resolution rules, namely the unbiased rule and the proposed biased rule, in terms of feasibility of invoking the cascade phenomenon in spectrum contentions under various conditions in CR networks.

\subsection{Simulation Setup}

Topology. We simulate three typical lattices: coexisting BSs are placed on a honeycomb lattice $(d=3)$, a square lattice $(d=4)$ and a triangular lattice $(d=6)$, respectively. 
Self-coexistence window and inter-BS spectrum contention. In a network cell, the BS provides broadband access to secondary users according to a time schedule consisting of superframes. 802.22 provides the inter-BS synchronization mechanism for neighboring BSs to align their superframes. In 802.22, a superframe has 16 frames, and a self-coexistence window (SCW) is periodically scheduled in every frame for spectrum contention.

Service requirement. There are a total number of $N=$ 30 channels in our simulations. Every BS requires 10, 20 or 30 channels to satisfy the QoS of its admitted workload.

Neighboring BSs occupy disjoint sets of channels, and a BS claims a number of channels which is no more than its service requirement.

Primary user (PU) traffic generation. We assume that there is one primary transmitter per cell, and every primary transmitter randomly selects $X \in\left[0, N_{a}\right]$ channels to emit its signals, where $N_{a}$ is the number of PU's active channels. In most existing work, it is assumed that a primary transmitter follows a "busy/idle" traffic pattern on a licensed channel $[4,11]$. Hence, a "busy/idle" pattern is simulated for each primary transmitter: the busy period has a fixed length of $b$ timeslots, and the idle period follows an exponential distribution with a mean of $l$ frames. Thereafter, we will simply refer to the notation $\lambda_{e}=\frac{1}{l}$ as the primary transmission rate. Every $\mathrm{BS}$ is able to detect the signals from the primary transmitter in the same cell. A channel is considered "unavailable" when primary user signals are present in it. All secondary users (BSs) should vacate unavailable channels during the period of primary user transmission.

\subsection{Verification of Applicable Criteria in 802.22 Networks}

In Theorem 5, we propose applicable criteria for global and severe cascades in 802.22 networks, all of which have the form $k+C_{0} p_{0} \geq C_{1}$. Since $\mathrm{PU}$ traffic is generated in a "busy/idle" pattern, we have $p_{0}=b /(b+l)$. We choose appropriate $k, b$ and $\lambda_{e}$ such that $k+C_{0} p_{0}=C_{1}$ (if $k+C_{0} p_{0}$ is much greater than $C_{1}$, the global or severe cascade will be more likely to occur). We verify the applicable criteria for global cascades in a honeycomb lattice, a square lattice and a triangular lattice, respectively.

We conduct 100 repeated experiments for each type of lattice. In the honeycomb lattice case, $94 \%$ of the repeated experiments show a global cascade with $\bar{\chi}=9285.69$, where $\bar{\chi}$ is the average value of $\chi$ in repeated experiments. In the square lattice case, $97 \%$ of the repeated experiments show a global cascade with $\bar{\chi}=9842.05$. In the triangular lattice case, $99 \%$ of the repeated experiments show a global cascade with $\bar{X}=9921.02$.

\subsection{Phenomenon of Cascading Spectrum Con- tentions}

We measure the mean cluster size, $\chi$, by varying the following parameters: the degree $d$ of the lattice where BSs are placed, the number $k$ of pairwise contentions initiated by a contention source, and the PU traffic pattern (the number $N_{a}$ of PU's active channels and the primary transmission rate $\lambda_{e}$ ).

Impact of lattice degree. In this set of simulations, we fix $r=k / d=1$. In the honeycomb lattice case $(d=3)$, the mean open cluster size is the smallest while the triangular

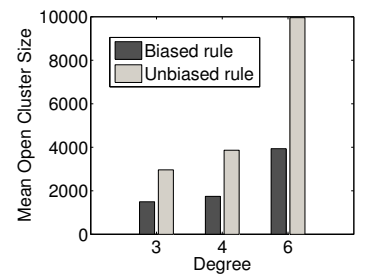

Figure 2: Mean open cluster size vs. lattice degree.

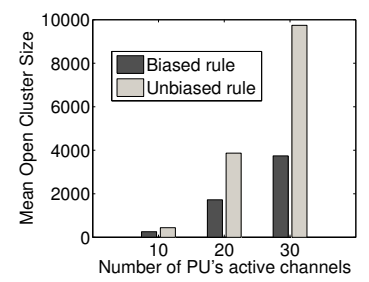

Figure 4: Mean open cluster size vs. number of PU's active channels.

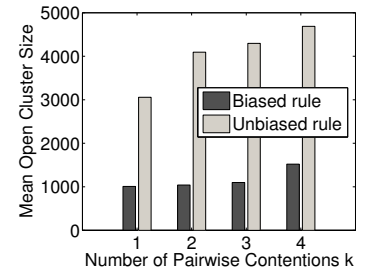

Figure 3: Mean open cluster size vs. number of pairwise contentions.

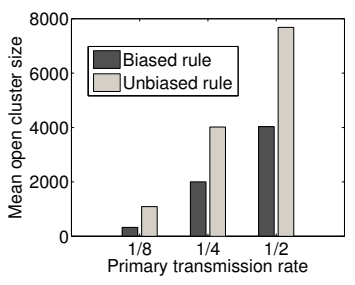

Figure 5: Mean open cluster size vs. primary transmission rate. lattice $(d=6)$ shows the largest mean open cluster size (Figure 2). These results coincide with the previous conclusion, with $r=k / d$ fixed, there is a positive correlation between $\chi$ and $d$.

Impact of number of pairwise contentions. As is shown in Figure 3, the more pairwise contentions initiated by a contention source, the larger the mean open cluster size.

Impact of number of PU's active channels. When $N_{a}=10$, every BS is able to occupy ten channels to satisfy its channel requirement, and it will become starving once some of its occupied channels are reclaimed by primary transmitters. When the primary transmitter is active on every channel $\left(N_{a}=30\right)$, a BS is prone to the lack of spectrum and entering the contention initiation phase, and thus the cascade phenomenon appears as expected under the unbiased rule and almost every BS belongs to the unique open cluster (Figure 4).

Impact of the primary transmission rate. When the primary transmitter is active with a high primary transmission rate $(\lambda=1 / 2)$, a $\mathrm{BS}$ is prone to the lack of spectrum and entering the contention initiation phase, and thus the cascade phenomenon appears as expected under the unbiased rule. When the primary transmission rate is low, the effect of cascading spectrum contentions is not obvious under both contention resolution rules, since the primary user releases channels in most time such that most of BSs can be satisfied.

\section{CONCLUSION}

In this paper, we systematically studied the CR networks' resilience to the cascading spectrum contentions. We model the cascading spectrum contention problem as a site percolation process in the underlying network graph. Under the percolation-based framework, we use the mean open cluster size $\chi(p)$ to measure the magnitude of the cascade of spectrum contentions and mathematically relate the starv- 
ing probability $p$ to $\chi(p)$. We identify the four parameters $d, k, \lambda$ and $p_{0}$, and establish the relationship between $p$ and these parameters, through which we obtain the criteria to detect global or severe cascades. In addition, we further improve the obtained criteria to be computationally applicable, and apply them to the 802.22 networks for detecting global or severe cascades. Finally, we propose a biased spectrum contention protocol that allows coexisting CR networks to prevent the cascading spectrum contentions. To the best of our knowledge, this is the first work to study the cascading spectrum contention problem in self-coexistence of CR networks.

\section{Acknowledgements}

This work was partially supported by National Natural Science Foundation of China under grant numbers 61201245, 61073155 and 61001075 .

\section{REFERENCES}

[1] M. Aizenman and C. M. Newman. Tree Graph Inequalities and Critical Behavior in Percolation Models. Journal of Statistical Physics, 36(1/2):107-143, 1984.

[2] O. Dousse, F. Baccelli, and P. Thiran. Impact of Interferences on Connectivity in Ad Hoc Networks. IEEE Transactions on Networking, 13(2):425-436, April 2005.

[3] O. Dousse, P. Mannersalo, and P. Thiran. Latency of Wireless Sensor Networks with Uncoordinated Power Saving Mechniasm. Proc. of ACM MobiHoc, pp. 109-120, 2004.

[4] S. Geirhofer, L. Tong, and B. Sadler. Cognitive Medium Access: Constraining Interference Based on Experimental Models. IEEE Journal on Selected Areas of Communications, 26(1):95-105, Jan. 2008.

[5] D. Grandblaise and W. Hu. Adaptive On Demand Channel Contention. IEEE docs: IEEE 802.22-08/0078r0, Feb. 2009.

[6] D. Grandblaise and W. Hu. Inter Base Stations Adaptive On Demand Channel Contention for IEEE
802.22 WRAN Self Coexistence. IEEE docs: IEEE 802.22-07/0024r0, Jan. 2007.

[7] G. Grimmett. Percolation. New York: Springer, second edition, 1999.

[8] W. Hu. Frame-based On-demand Spectrum Contention Protocol-the Specifications. IEEE docs: IEEE 802.22-07/0024r0, Jan. 2009.

[9] W. Hu. On Demand Channel Contention: A Scalable and Fair Spectrum Sharing Protocol for TVWS Coexistence. IEEE docs: IEEE 802.22-08/0078r0, July 2009.

[10] W. Hu, M. Gerla, G.A. Vlantis, and G. J. Pottie. Efficient, Flexible, and Scalable Inter-network Spectrum Sharing and Communications in Cognitive IEEE 802.22 Networks. Proc. of CogART 2008, pp. $1-5,2008$.

[11] S. Huang, X. Liu, and Z. Ding. Optimal Transmission Strategies for Dynamic Spectrum Access in Cognitive Radio Networks. IEEE Transactions on Mobile Computing, 8(12):1636-1648, Dec. 2009.

[12] IEEE 802.22 Working Group. http://www. ieee802.org/22/.

[13] H. Kim and K. G. Shin. Asymmetry-Aware Real-Time Distributed Joint Resource Allocation in IEEE 802.22 WRANs. Proc. of IEEE INFOCOM, March 2010.

[14] Z. Kong and E. M. Yeh. Distributed Energy Management Algorithm for Large-scale Wireless Sensor Networks. Proc. of ACM MobiHoc, Sept. 2007.

[15] Z. Kong and E. M. Yeh. Connectivity and Latency in Wireless Networks with Unreliable Links. Proc. of IEEE INFOCOM, April 2008.

[16] Z. Kong and E. M. Yeh. On the Latency of Information Dissemination in Mobile Ad Hoc Network. Proc. of ACM MobiHoc, pp. 109-120, 2008.

[17] Z. Kong and E. M. Yeh. Resilience to Degree-dependent and Cascading Node Failures in Random Geometric Networks. IEEE Transactions on Information Theory, 56(11), pp. 5533-5546, 2010. 